TITRE: INTÉGRATION DE LA MULTIMODALITÉ DANS LES PROJETS D'INNOVATION DE STAGIAIRES EN FORMATION

Auteur(s): Nathalie Lacelle, Ph.D., UQAM et Amal Boultif, ChARgÉE de COURS, UQAM

PubliCATION: LA RECHERCHE-ACTION ET LA RECHERCHE-DÉVELOPPEMENT AU SERVICE DE LA LITTÉRATIE.

PAGES: $68-96$

DiRecteurs: ISABElle CARIgnan, MARIE-Christine BEAUdRY ET FrançoIs LAROSE

ÉdiTEUR: LES ÉdITIONS DE L'UNIVERSITÉ DE SHERBROOKE, 2016.

ISBN: 978-2-7622-0354-7

URI: HTTP://HDL.HANDLE.NET/11143/8814

DOI: HTTP://DX.DOI.ORG/10.17118/11143/8814 


\section{Intégration de la multimodalité dans les projets d'innovation de stagiaires en formation}

Nathalie Lacelle, Ph.D., UQAM

Amal Boultif, chargée de cours, UQAM

Résumé : L'article présente une recherche-action-formation menée auprès de seize étudiants en formation pratique qui ont eu à intégrer des pratiques multimodales à leur projet d'innovation pédagogique. Nous décrivons et analysons les modalités d'accompagnement des stagiaires impliqués afin de déterminer comment ces dernières ont permis le transfert de connaissances en recherche dans la pratique enseignante grâce à un accompagnement universitaire inspiré du nouveau paradigme de la multimodalité. Nous nous appuyons essentiellement sur les bilans réflexifs des stagiaires qui consignent leurs analyses quant à l'intérêt et à l'efficacité du recours à des pratiques d'enseignement multimodales pour résoudre des difficultés d'apprentissage disciplinaires de leurs élèves lors des stages. En ce sens, les connaissances produites sont à la fois méthodologiques et praxéologiques. Enfin, nous proposons des pistes de réflexion sur l'apport de la littératie médiatique et des TIC sur l'enseignement des disciplines et sur la manière dont la recherche peut aider les enseignants en formation à innover afin de développer des pratiques d'enseignement en phase avec les modes de communication modernes.

Mots-clés : littératie médiatique multimodale, projets d’innovation, stagiaires, formation initiale. 


\section{Introduction}

Les recherches récentes visant à renouveler les approches didactiques en lecture/écriture sur des supports variés, intégrant à différents degrés l'image, le texte et le son, soutiennent la nécessité de passer du paradigme de la littératie classique (monomodale) à celui de la littératie médiatique ${ }^{1}$ (multimodale) en éducation (Lebrun, Lacelle et Boutin, 2012a, 2012b). Or, un tel changement dans les conceptions et les pratiques des enseignants passe assurément par l'intégration de ces nouvelles approches dans la formation universitaire et la formation continue. Dans le cadre de cet article, qui remet en question le transfert de la recherche dans la formation supérieure, nous présenterons la mise en œuvre d'une recherche-action-formation auprès de seize étudiants ${ }^{2}$ en formation pratique qui ont eu à intégrer des pratiques multimodales au sein de leurs projets d'innovation pédagogique. Nous décrirons et analyserons les modalités d'accompagnement des stagiaires impliqués et nous tenterons de déterminer comment ces dernières ont permis le transfert de connaissances en recherche dans la pratique enseignante. Nous nous appuierons essentiellement sur les bilans réflexifs des stagiaires qui consignent leurs analyses quant à l'intérêt et à l'efficacité du recours à des pratiques d'enseignement multimodales pour résoudre les difficultés d'apprentissage disciplinaires de leurs élèves lors des stages. En ce sens, les connaissances produites seront à la fois méthodologiques et praxéologiques. Enfin, nous proposerons des pistes de réflexion sur l'apport de la multimodalité, de la littératie médiatique et des technologies de l'information et de la communication (TIC) à l'enseignement des disciplines et sur la manière dont la recherche peut aider les enseignants en formation à innover afin de développer des pratiques d'enseignement en phase avec les modes de communication modernes. Deux questions fondamentales sous-tendent cette recherche: comment la recherche-action-formation favorise-t-elle le transfert de connaissances issues de la recherche à la pratique de stagiaires en formation? Quelles sont les incidences de ce transfert sur le développement de nouvelles pratiques didactique?

\footnotetext{
1. «Le concept de «littératie médiatique» (media literacy), auquel on accole le qualificatif de « multimodale » se répand de plus en plus, particulièrement en français. II s'agit d'une acception qui recoupe à la fois l'« éducation aux médias » (media éducation) et les usages multimodaux des médias dans des contextes diversifiés d'enseignement et d'apprentissage, en misant de façon spécifique sur les compétences en lecture/réception et en écriture/production avec les nouveaux médias, donc des compétences « textuelles » d'un nouveau genre.
}

2. Le stage IV s'inscrit dans le cadre du baccalauréat en enseignement secondaire. Ce stage d'internat à l'école secondaire s'effectue sous la supervision d'un enseignant associé. Le stagiaire y prend en charge toutes les responsabilités d'une tâche d'enseignement à des groupes classes et l'encadrement d'activités éducatives. Il s'agit essentiellement d'une période d'entrainement et d'expérimentation de sa future profession. 


\section{Problématique}

\section{Les enseignants et les TIC}

La littératie médiatique multimodale (LMM), exigeant très souvent la manipulation des TIC par les enseignants, il convient d'y intégrer des compétences technologiques. Or, une enquête réalisée auprès de 2065 futurs enseignants en stage au Québec portant sur une appréciation de chaque composante de la compétence professionnelle à intégrer les TIC (Villeneuve et coll., 2012) révèle que la compétence à « communiquer à l'aide d'outils multimédias variés » est la moins maitrisée. Une revue des recherches sur les pratiques enseignantes effectuée par Bussière et Gluszunski (2004) laisse supposer que ce n'est pas tant la disponibilité des TIC, mais la qualité et le type d'utilisation qui constituent un facteur-clé de l'apprentissage. Plusieurs chercheurs canadiens (Gauthier, 2006; Barrette, 2004; Bibeau, 2003) voient dans l'élaboration et l'expérimentation de scénarios didactiques en littératie médiatique la solution à une véritable mise en place de pratiques enseignantes intégrant les TIC. D’autres chercheurs anglais, américains et australiens (Walsh, 2008; Kress, 2003; Jewitt, 2002; Kress et coll., 2001; Kress et Van Leeuwen, 2001) insistent aussi sur la création en recherche de designs pédagogiques originaux utilisant des modes d'expression différents pour enrichir les curriculums actuels. Il apparait clairement que les enseignants ont besoin d'être accompagnés dans la création de scénarios didactiques qui s'appuient sur des pratiques multimodales, l'usage des TIC, et qui mobilisent chez les élèves des compétences en LMM.

Dans la pratique éducative, ceux (les enseignants) qui adoptent une plus large perspective sur les littératies et sur ce qui compte comme texte ne s'appuient pas uniquement sur la lecture de manuels scolaires et la production de copies écrites, mais ils amènent les élèves à voir comment les messages sont communiqués par plusieurs moyens, y compris les représentations dramatiques, les posters, les photos, les graphies, les images, les sites web, les CD-ROM, les DVDs, les jeux vidéos, la production vidéo et les autres outils des technologies numérisées (Street et coll. 2009; Cope et Kalantzis, 2000). (Dagenais, 2012, p. 25)

Les TIC se révèlent donc efficaces lorsqu'elles s'intègrent à une didactique diversifiée qui articule finement les activités d'apprentissage disciplinaires, les approches pédagogiques et les supports multimodaux. 


\section{Les TIC et la multimodalité en formation universitaire}

La présente recherche s'intéresse principalement aux composantes suivantes: communiquer à l'aide d'outils multimédias variés (3); utiliser efficacement les TIC pour rechercher, interpréter, communiquer de l'information et pour résoudre des problèmes (4); aider les élèves à s'approprier les TIC, à les utiliser pour faire des activités d'apprentissage, à évaluer leur utilisation de la technologie et juger de manière critique les données recueillies sur les réseaux (6). Le recours à la multimodalité (l'image, la vidéo, les cartes conceptuelles, les fichiers audios) par l'usage des technologies est ponctuellement recommandé, mais jamais dans une perspective de recours à la LMM pour s'approprier des contenus disciplinaires.

\section{Les objectifs de la recherche}

Dans le cadre de cette recherche, nous nous intéressons aux pratiques d'enseignement-apprentissage qui concernent l'usage de la multimodalité (combinaisons de modes sémiotiques et de médias) à travers des scénarios didactiques qui intègrent les multitextes (blogues, BD, vidéos, jeux vidéos, etc.). Nous proposons des outils méthodologiques (recherche) et didactiques (pratique) pour favoriser le recours à la multimodalité en enseignement des disciplines ${ }^{4}$ et aux compétences en LMM pour l'apprentissage. L'objectif général de cette recherche-action-formation vise à documenter le développement de pratiques multimodales d'enseignement-apprentissage de contenus disciplinaires par des enseignants stagiaires. Ces derniers devaient identifier un problème de départ, anticipé ou constaté chez leurs élèves, à la suite duquel ils devaient bâtir un projet pédagogique innovant intégrant la multimodalité et les TIC.

\section{Le modèle du TPaCK de Koehler et Mishra et la grille en LMM de Lebrun, Lacelle et Boutin}

Deux grilles de lecture, issues du cadre théorique des littératies médiatiques multimodale en contexte pédagogique, nous ont servi d'outils d'analyse des projets d'innovations des stagiaires sous deux angles complémentaires : celui des usages de la technopédagogie au moyen du modèle TPaCK

3. La multimodalité se caractérise par la présence de différents modes iconiques, linguistiques, gestuels et auditifs. Elle est toujours au moins à deux niveaux : premièrement, on retrouve une juxtaposition ou combinaison de différents modes et, deuxièmement, ces mêmes modes ont une nature multimodale (une séquence vidéo, par exemple, comprend des images animées, une gestuelle et des sons, les trois étant livrés symbiotiquement). C'est l'articulation des modes d'expression qui fait en sorte que le texte multimodal prend forme; il en va de même pour la combinaison de médias qui exigent du lecteur qu'il tisse les liens entre les informations recueillies à partir de divers documents multimodaux (Kress, 2010; Foucher, 1998).

4. Au Québec, le guide utilisé auprès des enseignants du préscolaire-primaire et du secondaire date de 2001 (MEQ). II comporte douze compétences dont la huitième s'intitule «Intégrer les technologies de l'information et des communications aux fins de préparation et de pilotage d'activités d'enseignement- apprentissage, de gestion de l'enseignement et de développement professionnel » et est constituée de six composantes (MEQ, 2001). 
(Mishra et Koehler, 2006) et celui de la multimodalité au moyen d'une grille des compétences en LMM (Lebrun, Lacelle, Boutin, 2012a, 2012b).

\section{Le modèle du TPaCK de Koehler et Mishra}

Le modèle du TPaCK offre une analyse pertinente des usages des TIC en contexte d'enseignement-apprentissage (Schmidt et coll. 2009). Ses concepteurs (Mirsha et Koehler, 2006 et 2009) le situent dans le prolongement du modèle de Shulman (1986) qui ne comprenait que deux éléments de départ dans le domaine de l'enseignement-apprentissage : le savoir sur les contenus à enseigner et le savoir sur la pédagogie qui sous-tend ces enseignements (Pedagogical content knowledge). Les travaux de Herring, Mishra et Koehler $(2016)^{5}$ ont enrichi ce cadre en y intégrant la technologie (Technical Pedagogical Content Knowledge). Ce modèle intégrateur veut contrer la vision uniquement instrumentale qu'ont beaucoup d'enseignants usagers des TIC.

La figure 1 présente les combinaisons considérées dans le modèle d'intégration des savoirs technologiques aux savoirs pédagogiques et de contenu élaboré par Koehler et Mirsha 2008).

5. Voir aussi le site du TPACK http://www.tpack.org/ crée par Mirsha et Koehler qui regroupe tous les travaux du groupe des deux chercheurs.

6. PK+CK : mise en œuvre conjointe des savoirs pédagogiques et des contenus qui sont les connaissances nécessaires pour l'enseignement d'un certain contenu disciplinaire.

PK+TK : l'intersection technologie / pédagogie renvoie à la compréhension des différentes façons dont la technologie peut être utilisée dans un contexte pédagogique.

CK+TK : les savoirs de contenu disciplinaire ajoutés aux savoirs technologiques permettent à l'enseignant d'avoir des représentations de la manière dont le contenu disciplinaire peut être soutenu ou approfondi à l'aide de la technologie.

TK+PK+CK : désigne la convergence des trois domaines en interaction. Nous avons là le cœur du modèle TPaC; il présente « les habiletés à développer des expériences d'apprentissage signifiantes pour les élèves en intégrant de façon dynamique les aspects relevant des contenus disciplinaires, de la pédagogie et de la technologie». 
Figure 1 : Modèle TPaCK

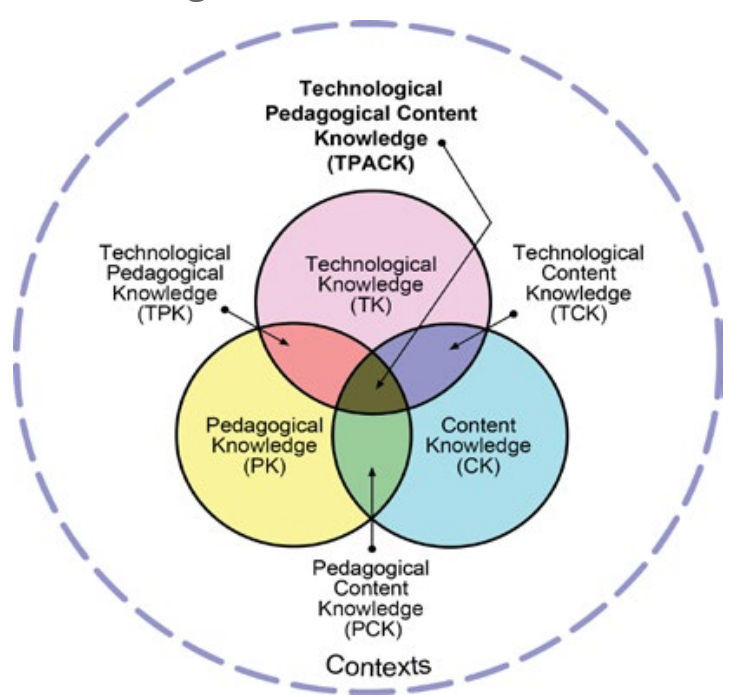

Image reproduite avec l'autorisation des éditeurs, (c) 2012 by tpack.org

Pour le présent article, nous nous sommes servies du modèle TPaCK $\mathrm{T}^{7}$ puisque nos questions de recherche étaient centrées sur les modalités d'accompagnement des stagiaires impliqués dans ce projet et sur les aspects praxéologiques qui touchent au transfert de connaissances en recherche dans la pratique enseignante.

\section{La grille en LMM de Lacelle, Lebrun, Boutin, Richard et Martel}

La grille transdisciplinaire en LMM (Lacelle et coll., 2015) vise essentiellement l'intégration de pratiques multimodales dans l'enseignement et l'apprentissage des disciplines. L'espace limité de cet article nous contraint à ne soumettre qu'une description sommaire de la grille de compétences en litmedmod ${ }^{8}$. Cette grille explicite les compétences cognitives et affectives (ex : décoder, comprendre et intégrer un message multimodal), les compétences pragmatiques générales (ex : reconnaitre et analyser les contextes de réception et/ou de production d'un message multimodal), les compétences sémiotiques générales (ex : reconnaitre/analyser/communiquer les signes ou symboles du message multimodal), la compétence modale spécifique (ex : reconnaitre/analyser/utiliser les ressources sémiotiques propres au mode visuel), la compétence multimodale (ex: reconnaitre/analyser/appliquer la simultanéité d'utilisation des codes, des modes, des langages et leurs modalités).

7. Figure 1

8. Pour consulter la grille complète : litmedmod.ca, onglet « outils». 


\section{La méthodologie : une recherche-action-formation en contexte de formation pratique des maitres}

Le paradigme de la multimodalité étant peu connu des enseignants ainsi que les compétences en littératie médiatique nécessaires aux apprenants pour accéder aux savoirs disciplinaires, nous avons choisi de recourir à une démarche de recherche-action-formation qui accentue le processus de la formation dans la recherche. La recherche-action-formation s'inscrit dans le sens d'une recherche pédagogique (Van der Maren, 2002) dont l'objectif premier est la fonctionnalité des savoirs produits à la fois pour l'action en classe et pour la formation des maitres (Prud'homme, 2007). Le chercheur-formateur doit donc considérer des enjeux à la fois scientifiques et pédagogiques dans l'élaboration du devis méthodologique.

\section{Les participants à la recherche}

Le projet de recherche a été initié et piloté par la chercheuse principale, Nathalie Lacelle, membre fondateur du groupe de recherche en littératie médiatique et multimodale LMM ${ }^{9}$, elle-même responsable d'un premier groupe de stagiaires $(\mathrm{N}=8)$. Une deuxième superviseure d'un deuxième groupe $(\mathrm{N}=8)$ avait la responsabilité de recueillir les rapports de stages et les documents utiles au projet. Une stagiaire doctorale ${ }^{10}$, Amal Boultif, spécialiste dans les usages pédagogiques des TIC, de la technologie à des fins d'enseignement et d'apprentissage de la lecture et de l'écriture, membre du groupe de recherche en Littératie Médiatique Multimodale (LMM), a dispensé aide et formation aux stagiaires et a participé à la collecte et à l'analyse des données.

Les sujets de la recherche, au nombre de seize, ont été recrutés dans les deux groupes composant les étudiants des séminaires des deux superviseures de stage, qui avaient la charge de former, d'encadrer et d'évaluer les apprentissages des futurs enseignants pour leur stage final d'internat en enseignement au secondaire. Au cours de ce stage, les stagiaires devaient prendre en charge toutes les responsabilités d'une tâche d'enseignement pendant douze semaines. Pour les fins de la recherche, et dans le but d'améliorer le développement de leur compétence technodidactique, ces derniers devaient élaborer un projet d'innovation pédagogique intégrant la multimodalité et l'expérimenter dans leurs classes respectives, soit de la $2^{e}$ à la $4^{e}$ secondaire. Des seize projets d'innovation, dix ont été jugés suffisamment complets pour les analyses de la recherche. Nous n'avons donc considéré, au final, pour la présente étude, que les projets finalisés et accompagnés des prétests ${ }^{11}$, des rapports du plan d'innovation et des bilans réflexifs qui s'y rattachent.

9. Groupe de recherche en littératie médiatique multimodale : http://www.litmedmod.ca/

10. Stage II du doctorat en éducation à l'Université du Québec à Montréal.

11. Nous explicitons ce point dans notre discussion. 


\section{Le design de la recherche}

Notre recherche a progressé en trois temps : le premier nous a permis de dispenser une formation aux stagiaires; le second, de documenter le travail des stagiaires et leurs usages de la multimodalité dans leurs classes durant la mise en œuvre du projet; enfin, le troisième, de recueillir les projets finalisés et les commentaires des enseignants stagiaires. Nous nous sommes concentrées, pour la présente recherche, à observer les modalités de mise en place de projets d'innovation pédagogiques en lien avec plusieurs disciplines du secondaire ${ }^{12}$ : cinq projets en français langue d'enseignement, trois projets en univers social et deux projets en mathématiques et sciences et technologie. Nous avons documenté les contenus des projets des stagiaires ( $\mathrm{N}=10)$ ainsi que leurs usages des TIC et des divers médias sollicités pour élaborer, mettre en œuvre, enseigner, consigner et conserver les traces des séquences d'enseignement apprentissage. Voici les principaux éléments qui composent le dispositif de la recherche ${ }^{13}$ :

- la formation dispensée par l'entremise des séminaires;

- les instruments de collecte de données : les questionnaires, les projets d'innovation et leurs plans de progression, les vidéos de présentation des projets et les journaux des chercheuses;

- le type de données (qualitatives et quantitatives) attendues et recueillies : bilans réflexifs des stagiaires et des chercheuses, réponses aux questionnaires (pratiques déclarées), présentations orales filmées des stagiaires (pratiques effectives) et bilans du stage;

- les instruments d'analyse prévus dans notre cadre théorique : le modèle TPaCK et la grille de compétences en LMM;

- indices de compréhension et d'intégration de la LMM, du TPaCK et portraits évolutifs des sujets de la recherche.

12. En conformité avec le programme de formation de l'école québécoise pour le secondaire et avec l'approche par projet qui recommande le développement de compétences transversales et disciplinaires en lien avec les usages des TIC et de la LMM.

13. Voir figure 2: design de la recherche. 
Figure 2 : Design de la recherche ${ }^{14}$

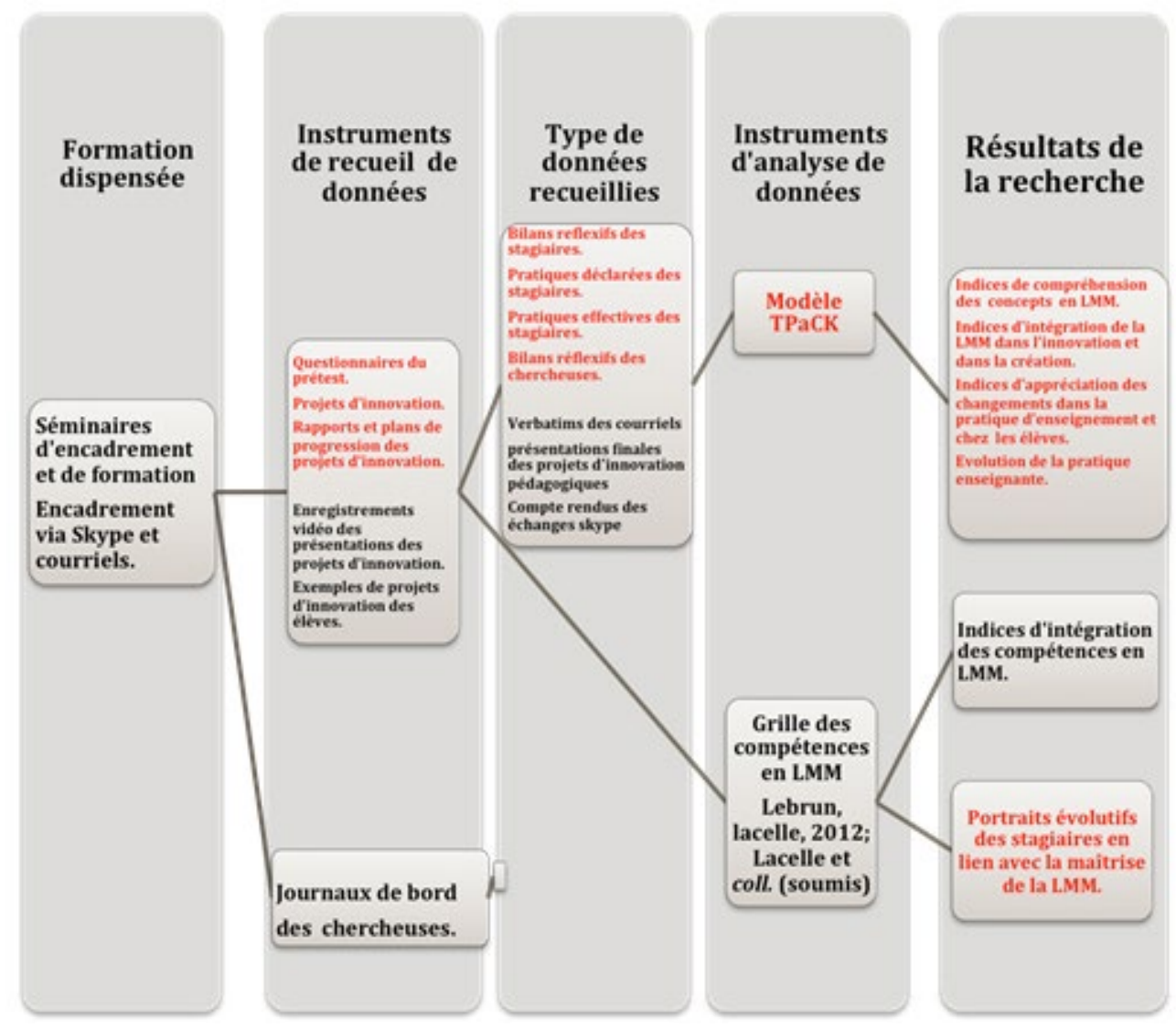

La phase de formation et modalités de suivis des enseignants stagiaires

L'équipe de chercheuses a conçu un document d'accompagnement (outils de collecte de données) pour les stagiaires leur permettant, dans un premier temps, d'évaluer les besoins de leurs élèves puis, dans un second temps, de déterminer les objectifs et les moyens pédagogiques et technologiques à mettre en place susceptibles d'y répondre.

L'annexe 2 présente un tableau qui fait la synthèse de la démarche d'encadrement et de formation de stagiaires, soit le nombre de stagiaires, le type d'encadrement et les objectifs des rencontres (séminaires et rencontres diverses), le nombre d'heures allouées à la formation et la nature de l'aide apportée aux stagiaires par l'équipe de recherche.

14. En rouge apparaissent les éléments développés dans le présent article. 


\section{Le traitement des données}

Les données recueillies correspondent à trois temps de notre recherche : le temps (1) qui précède la mise en œuvre pratique des projets d'innovation, qui touche plus particulièrement à la formation des stagiaires, et qui documente spécifiquement l'étendue des connaissances préalables des stagiaires et leurs compétences en lien avec la multimodalité; le temps (2) qui documente le cheminement des stagiaires et leur appropriation de la multimodalité et de ses pratiques effectives en contexte d'enseignement-apprentissage; enfin, le temps (3) qui documente la finalisation de projets d'innovation pédagogiques et l'évolution de la pratique de la multimodalité en contexte pédagogique chez les enseignants stagiaires. Comme les réponses aux questionnaires comportent des données chiffrées (questions fermées) et des questions à développement (questions ouvertes), cela implique un traitement qualitatif et quantitatif des données. Le codage des questions ouvertes ainsi que les commentaires du bilan réflexif figurant dans le rapport du plan de progression des stagiaires ont été saisis puis traités pour une analyse de contenu sémantique.

Le tableau 1 décrit les différents instruments de collecte de données, les moments documentés de la recherche (pré ou post expérimentation des stagiaires), le type de données qualitatives et quantitatives recueillies en fonction des objectifs de la recherche.

Tableau 1 : recueil de données et modalités de traitement

\begin{tabular}{|c|c|c|}
\hline \multicolumn{3}{|l|}{ Traitement des données } \\
\hline $\begin{array}{l}\text { Instruments de recueil } \\
\text { des données }\end{array}$ & Moments documentés & Thématiques et catégories recensées \\
\hline Questionnaires prétest & $\begin{array}{l}\text { Avant la mise en place des projets d'inno- } \\
\text { vation pédagogiques. }\end{array}$ & $\begin{array}{l}\text { Usages et pratiques autodéclarées par les stagiaires sur le degré de } \\
\text { compréhension initial de la LMM, le degré de maitrise autodéclaré } \\
\text { des usages de la multimodalité et des outils technologiques. }\end{array}$ \\
\hline $\begin{array}{l}\text { Plans de progression } \\
\text { Courriels }\end{array}$ & $\begin{array}{l}\text { Pendant la réalisation et la mise en } \\
\text { œuvre des projets d'innovation pédago- } \\
\text { giques }\end{array}$ & $\begin{array}{l}\text { Intégration des catégories du TPaCK et en particulier de leurs inter- } \\
\text { sections à la pratique enseignante des stagiaires }{ }^{1}\end{array}$ \\
\hline $\begin{array}{l}\text { Rapports de stage et } \\
\text { bilans réflexifs }\end{array}$ & $\begin{array}{l}\text { Pendant et à la suite de la mise en pra- } \\
\text { tique des projets d'innovation pédago- } \\
\text { giques }\end{array}$ & $\begin{array}{l}\text { Compréhension de la multimodalité à la suite de la mise en œuvre } \\
\text { des plans d'innovation pédagogiques. } \\
\text { Usages disciplinaires de la multimodalité. } \\
\text { Évolution des stagiaires vers la multimodalité et stabilisation des } \\
\text { pratiques. }\end{array}$ \\
\hline
\end{tabular}




\section{L'analyse des données}

Pour une analyse approfondie de notre corpus de données, nous avons adopté une démarche propre aux méthodes des approches qualitatives, et cela, avec l'intention de répondre à nos questions et objectifs de recherche. Nous avons adopté le principe de l'analyse documentaire (L'Écuyer, 1990). La catégorisation des unités de sens s'appuie sur les catégories prédéterminées suivantes qui seront sous-divisées à leur tour selon les concepts de notre grille sur les compétences en LMM. Catégorie 1 : indices de compréhension des enseignants des concepts en LMM. Catégorie 2 : indices d'appropriation des enseignants de la grille de compétences en LMM. Catégorie 3 : indices d'intégration des enseignants de la multimodalité dans la création et l'expérimentation de dispositifs d'enseignement. Catégorie 4 : indices d'appréciation des enseignants quant aux changements de pratiques pédagogiques mis en place en multimodalité et leur perception des retombées sur leurs élèves. Les indices ont été regroupés selon les disciplines et mis ensuite en commun afin de voir de quelle façon les enseignants des différentes disciplines se situent dans leur utilisation de la multimodalité et des TIC. En fin de parcours, les résultats ont également été analysés selon le processus de l'intégration de l'innovation de Charlier (2005; 2009) en revenant sur : 1- les conditions ayant favorisé l'implantation de la LMM comme innovation; 2- l'évolution des enseignants vers la LMM (de l'intérêt à l'engagement); 3- la stabilisation des pratiques de LMM (en prenant principalement comme base les journaux de terrain des chercheuses).

\section{La discussion sur les résultats}

Nous présenterons les résultats d'analyse des réponses des stagiaires aux questionnaires du prétest et des verbatims des bilans du plan de progression (commentaires réflexifs). Nous procèderons ensuite à une réorganisation de ces données à la lumière de nos grilles d'analyse, soit le modèle TPaCK et la grille de compétences en LMM. Nous avons organisé nos données selon qu'elles relevaient de pratiques déclarées (prétest) ou de pratiques réelles (rapports du plan de progression et bilans réflexifs) pour en extraire les thèmes prédéterminés et émergents.

\section{Les pratiques déclarées de la maitrise de la multimodalité par les stagiaires ${ }^{15}$}

L'histogramme (figure 3) montre que la majorité des stagiaires se jugent de « moyens » à « bons » à faire usage de divers supports de la multimodalité. On note aussi que la production de messages textuels et iconiques fixes est perçue par les stagiaires comme la mieux maitrisée alors que la manipulation d'autres modes d'expression (les images séquentielles (BD) ou mobiles et les messages sonores) l'est beaucoup moins. Ceci se confirme dans le graphique suivant (figure 4) où l'on note que les combinaisons modales semblent poser problème aux stagiaires surtout dans le cas d'images séquentielles, mobiles ou avec du son.

15. Traitement quantitatif d'une partie des données du prétest : 4 questions adoptant la modalité de l'échelle de Likert. 
Figure 3 : Compétences multimodales déclarées sur divers supports

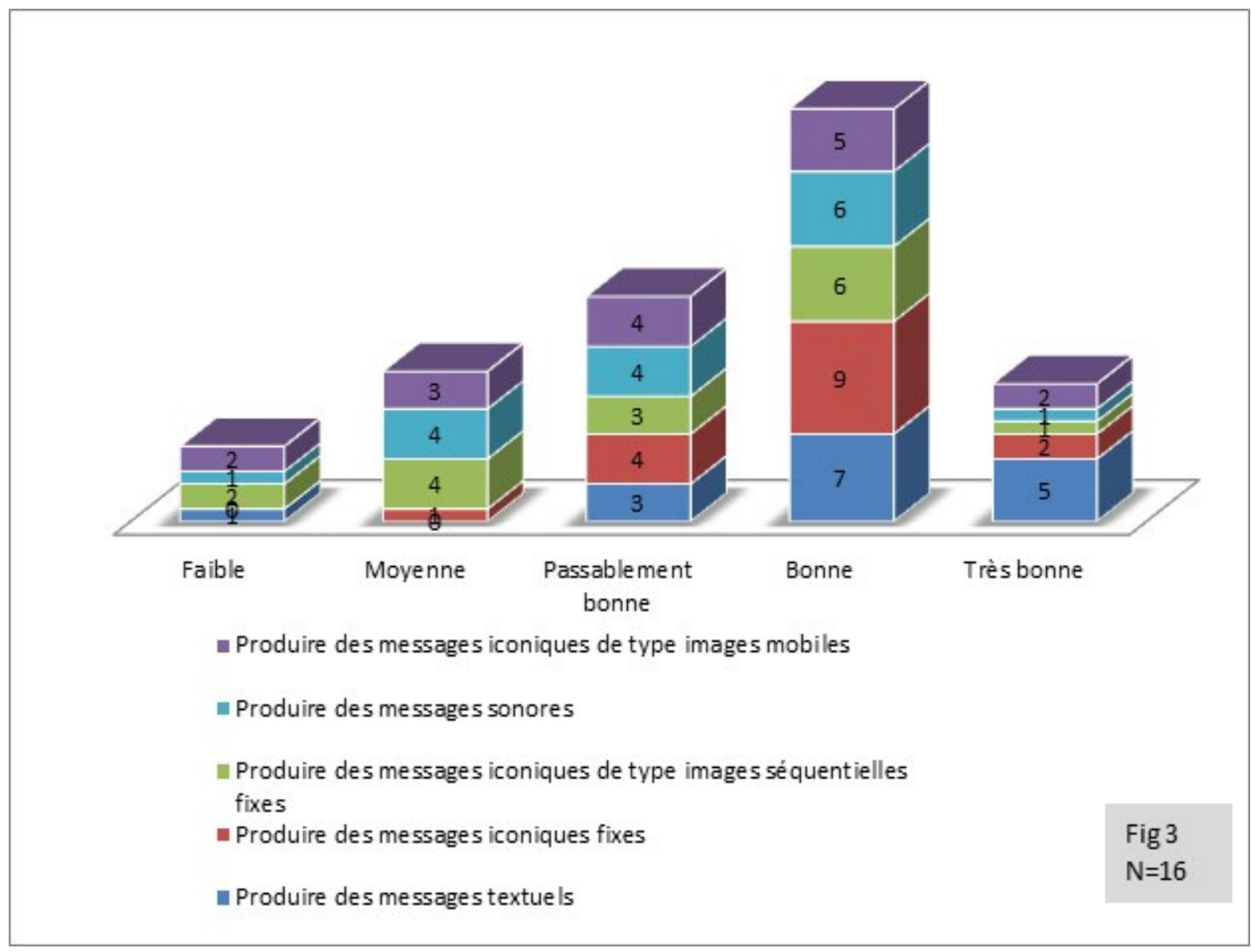


Figure 4 : Compétences déclarées à combiner la multimodalité en situation de production

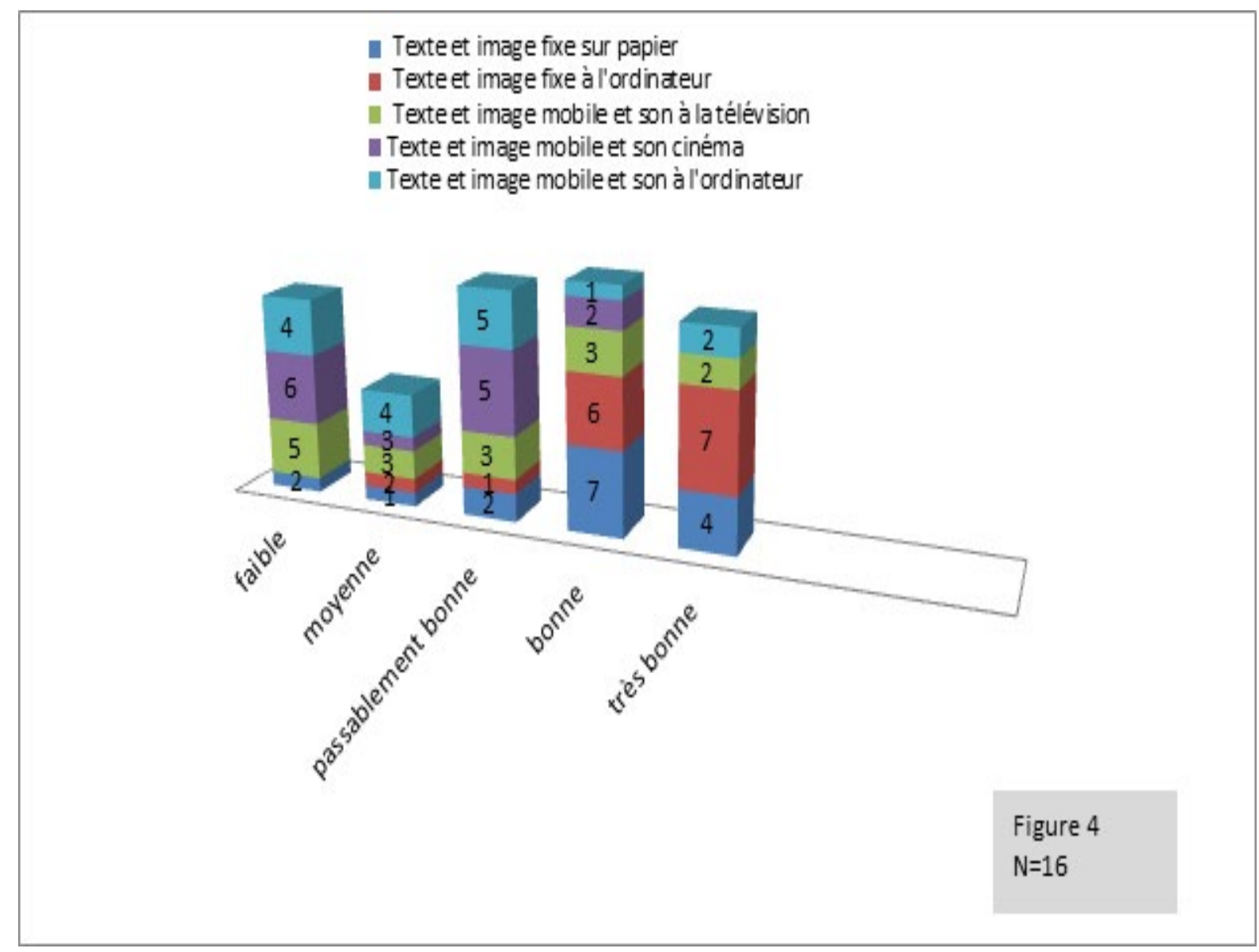

Les figures 5 et 6 s'appuient sur le traitement des données des questionnaires (catégorie des questions à développement) et illustrent l'importance accordée aux usages des outils technologiques chez les stagiaires (figure 5) ainsi que leur opinion sur l'utilité effective et l'apport de ces mêmes outils à leur pratique (figure 6). Les résultats montrent des indices très élevés d'appréciation des usages de la multimodalité chez les stagiaires qui cherchent à motiver leurs élèves qui baignent dans une culture multimodale et à varier leurs stratégies d'enseignement. Ce constat recoupe, encore une fois, les données des bilans réflexifs. 
Figure 5. Opinions des stagiaires sur l'importance de l'intégration d'outils technologiques à leur pratique pédagogique

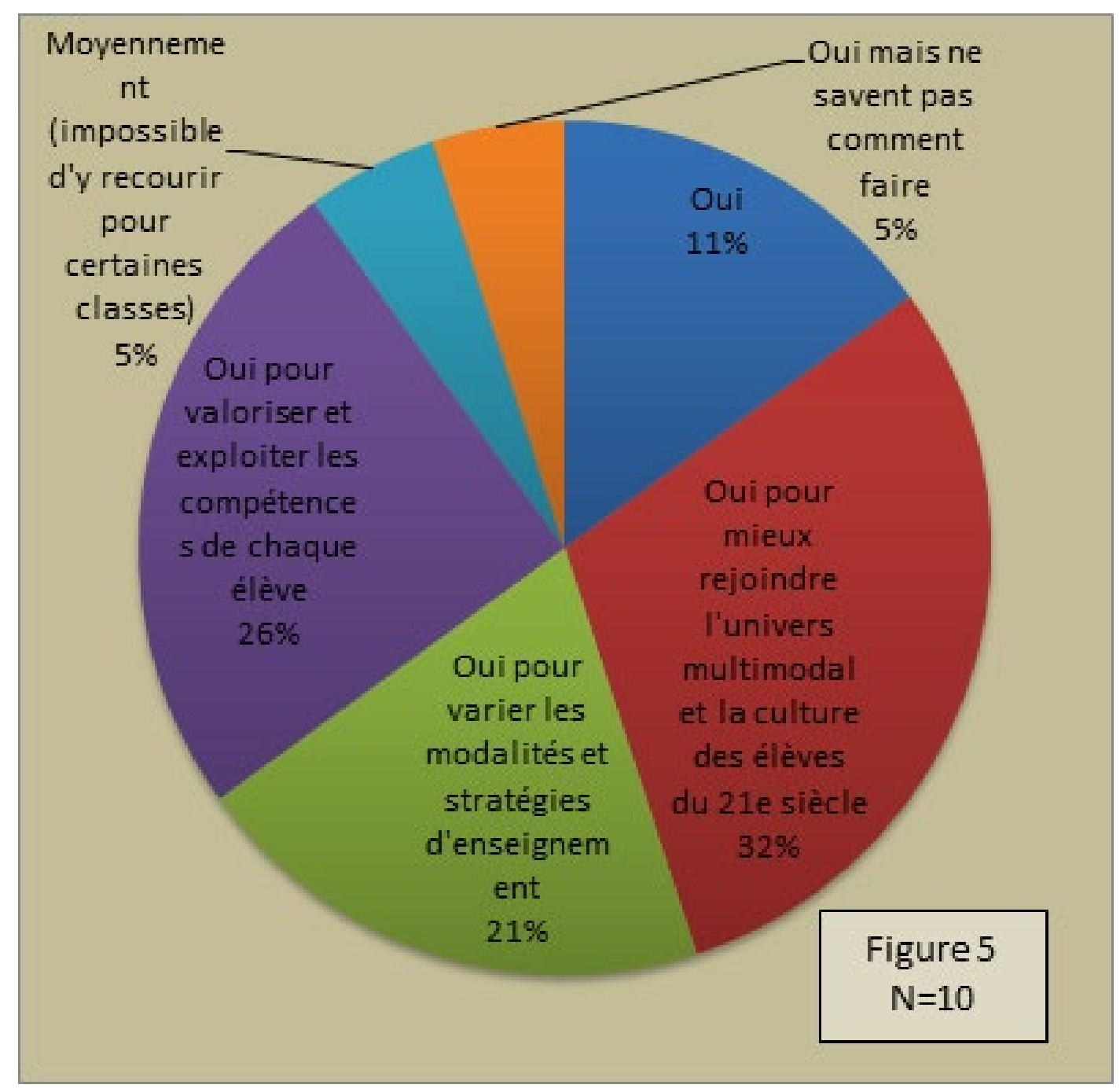


Figure 6: Opinion sur l'aide qu'apportent les supports multimodaux en enseignement-apprentissage

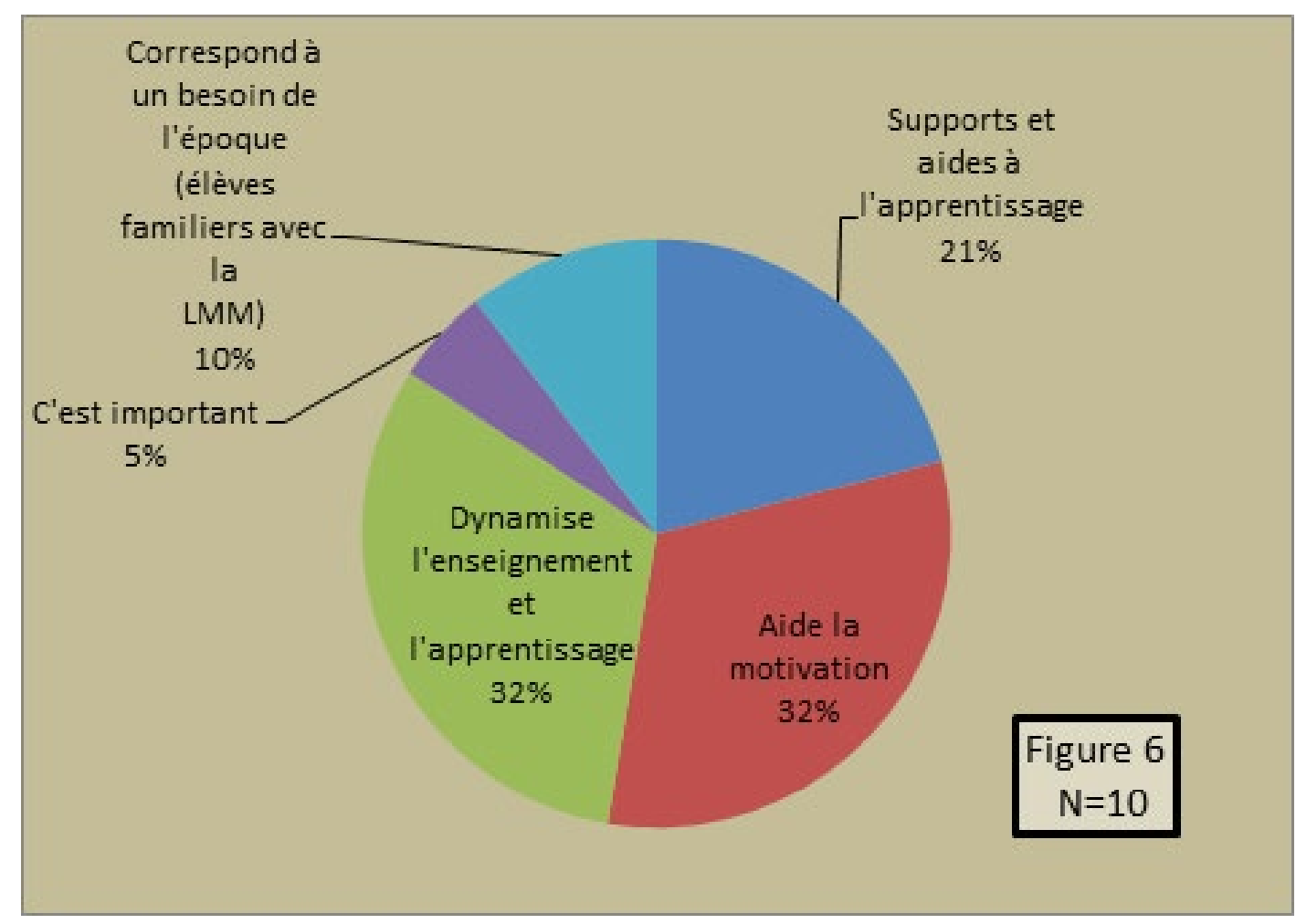

\section{La perception des retombées sur les élèves}

Dans leurs bilans, la majorité des dix stagiaires se déclarent satisfaits des objectifs atteints, mais ajoutent vouloir bonifier leurs projets afin de mieux combiner contenus, pédagogie et technologie. De plus, ils font état de changements chez les élèves, ce que des recherches sur les usages des TICE ont révélé, notamment la motivation et le rôle de la LMM dans l'appropriation du savoir (Loisier, 2011; Barette, 2004, 2005; Viau, 2005).

\section{Les indices de compréhension et d'appropriation des concepts en LMM}

Ces indices sont le fruit de la triangulation des éléments consignés par les étudiants dans leurs plans de progression du projet d'innovation, dans leurs bilans réflexifs (plan de progression du projet 
d'innovation ${ }^{16}$ ) et dans les réponses au questionnaire du prétes ${ }^{17}$. Notre analyse croisée montre une intégration très disparate de la LMM dans les projets d'innovation des dix stagiaires de notre échantillon. De plus, on remarque qu'il y a un écart entre les pratiques déclarées jugées assez moyennes et les usages réels qui sont faits de la LMM dans les projets de nos sujets qui révèlent une grande diversité d'usages.

Nous avons constaté, à la suite de l'analyse des projets, que cinq stagiaires ont eu plus de mal à arrimer tous les aspects de la multimodalité à leur pratique enseignante ${ }^{18}$.

J'ai pu développer mon habileté à utiliser différentes modalités pour la présentation de cours. C'est évident que j'appliquerais ce principe pour d'autres notions et activités. Cependant, je me rends compte, en complétant ce document, que je n'ai pas exploité les différentes modalités dans un objectif de développer des compétences chez les élèves par rapport à ces modalités. Donc, à l'avenir, j’essayerai de développer des compétences multimodales chez mes élèves. (Nora)

En effet, la multimodalité et les TIC sont surtout utilisées comme supports à l'enseignement. Les participants se sont limités à des usages des TIC pour l'enseignement (usage de logiciels et de supports et de ressources multimodales) sans nécessairement se pencher sur les compétences multimodales que les élèves doivent mobiliser pour s'approprier les savoirs disciplinaires. Et pour preuve, nous avons constaté l'absence d'évaluation des aspects relatifs à la multimodalité et à l'usage des TIC. Par opposition, nous remarquons que les cinq autres sujets, qui ont un score TPaCK élevé (figure 7) ont exploité tous les aspects collaboratifs et créatifs que peut offrir la multimodalité en enseignement et en apprentissage (les trois sphères du modèle y sont représentées) et ont, pour la plupart, intégré l'évaluation des usages de la multimodalité et des compétences en LMM à leurs projets.

Les indices d'intégration de la multimodalité dans la pratique des enseignants, selon le modèle TPaCK

Pour répondre à notre objectif de déceler des indices d'intégration de la multimodalité par les enseignants, nous avons établi un score TPaCK en partant des bilans réflexifs, des plans de présentation des projets d'innovation et des présentations finales des stagiaires. Nous avons accordé trois points pour les usages des trois dimensions en synergie dans les projets, deux points quand les seules dimensions étaient les savoirs pédagogiques et les savoirs relevant des contenus mis en œuvre et un seul point quand une seule dimension était présente.

Notre analyse des dix projets finalisés révèle une intégration partielle du modèle TPaCK par les stagiaires. En effet, cinq projets sur les dix révèlent des faiblesses quand il s'agit de considérer le

16. Voir annexe 3.

17. Administré à la fin des séminaires de formation.

18. Voir score TPaCK, supra. 
lien entre les trois pôles, soit les savoirs technologiques, les savoirs pédagogiques et les savoirs sur les contenus d'apprentissage. Ces faiblesses apparaissent quand on considère l'intersection entre le pôle technologique et le pôle pédagogique (TPK) et celui entre le pôle technologique et les contenus (TCK). Ce constat confirme les résultats des recherches antérieures qui font état des difficultés rencontrées par les enseignants à établir des liens entre les contenus à enseigner et les aspects pédagogiques et technologiques (Mishra et coll., 2009; Schmidt et coll., 2009). Le faible usage de dispositifs d'évaluation chez les enseignants croisant les compétences en LMM et en TIC (Béliveau, 2011) se confirme dans la présente recherche puisque deux seuls stagiaires y ont eu recours.

Figure 7 : Évaluation des projets d'innovation en fonction du score TPaCK.

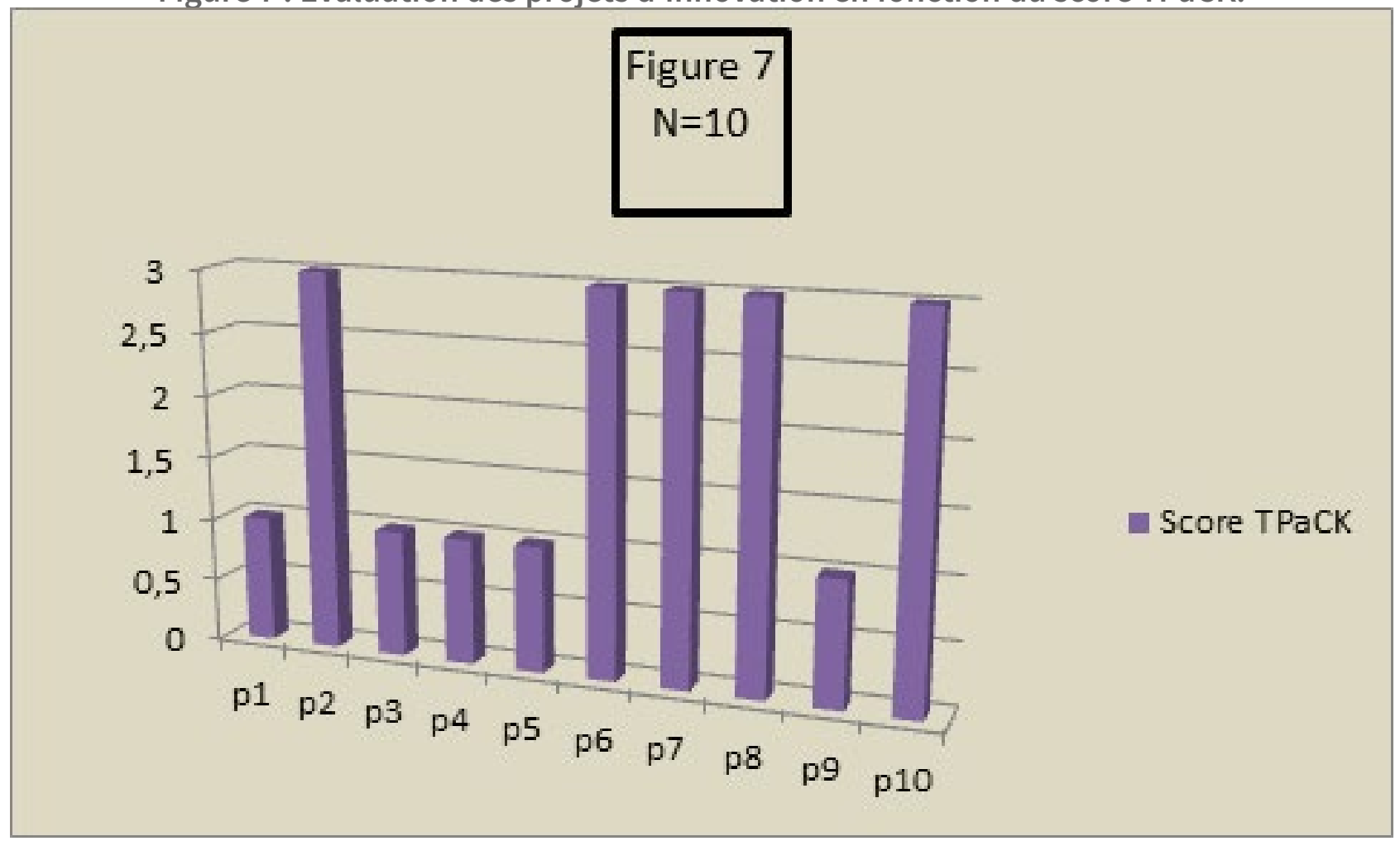

Bref, une forte tendance se dégage de nos observations : les stagiaires qui utilisent la technologie pour améliorer l'enseignement de leur discipline développent leur créativité et leur autonomie. C'est le cas des participants qui ont eu recours à des logiciels en ligne, ont réalisé un site web, ou encore ont produit une vidéo numérique d'apprentissage disciplinaire. Par exemple, un participant a réalisé un tutoriel sur support vidéo pour mieux aider ses élèves à comprendre un programme d'édition de textes en ligne.

\section{Indices d'évolution des pratiques des stagiaires}

La recherche-action-formation s'est avérée un déclencheur d'innovation des pratiques multimodales chez les participants. La formation reçue lors de séminaires universitaires a permis aux sta- 
giaires de vivre une véritable initiation à la multimodalité axée sur l'importance de créer des activités didactiques favorisant l'engagement des élèves et la mobilisation de compétences en LMM. Cela s'est confirmé dans plusieurs des projets que nous avons analysés.

Cette activité nous a permis d'échanger nos points de vue en plénière et ils (les élèves) ont aussi réalisé que le français n'est pas seulement de la grammaire, mais que tout peut devenir du français. Ils ont donc beaucoup apprécié les activités et le lien entre les élèves et moi est devenu plus fort à cause des différentes conversations que nous avons eues. (France)

Le dispositif de recherche mis en place nous a ainsi permis de réfléchir à l'impact de ce dernier sur les pratiques enseignantes des stagiaires. Nous avons aussi relevé que, de manière unanime, les stagiaires croient au potentiel motivant des TIC et au recours à des supports multimodaux en classe. Ils justifient ces usages par une pédagogie différenciée et variée appuyée par des méthodes d'enseignement-apprentissages diversifiées en lien avec la culture technologique des élèves. Ainsi, les stagiaires innovent en proposant des SAO (situation d'évaluation ouverte) adaptées aux usages de la LMM en contexte pédagogique.

J'avais envie de vivre une SAO (situation d'évaluation ouverte). Je trouve cette approche pédagogique intéressante pour la matière que j'enseigne. Je vois toutes les possibilités qui s'offrent à moi. (Paul)

Dans les projets, on note des indices de changement dans la pratique pédagogique des stagiaires qui s'appuient sur la diversification des outils technologiques des plateformes et des logiciels utilisés pour l'enseignement et l'apprentissage (figure 7). Les stagiaires citent les réseaux sociaux qui leur semblent utiles pour de futurs projets pédagogiques (Twitter, Wembly, etc.).

Concernant l'utilité de l'usage de la multimodalité en contexte pédagogique, notre analyse des bilans réflexifs et des questions à développement du questionnaire du prétest montre que presque tous les sujets expriment la nécessité d'en faire usage. Les arguments portent essentiellement sur le lien que la LMM permet d'établir avec les élèves comme moyen de les rejoindre (faire partie de leurs univers) et de les raccrocher (motiver) en faisant appel à leurs compétences informelles. Ainsi, à la question (2) du prétest:

"Comment l'utilisation des supports multimodaux peut-elle aider votre enseignement? », nous notons parmi les réponses:

Permettre aux élèves d'utiliser des supports avec lesquels ils sont familiers. (Paul)

Elle peut capter l'attention des élèves plus ou moins intéressés. Elle peut aussi permettre un intérêt chez quelques élèves. (Chantal) 
De plus, la multimodalité permettrait, selon les stagiaires, de varier les méthodes d'enseignement en diversifiant les stratégies, les outils et les activités proposées, et aussi de pouvoir s'adresser à tous les types d'apprenants en fonction de leurs intérêts, de leurs styles d'apprentissage et de leurs difficultés.

En allant rejoindre les diverses manières de comprendre chez les élèves (visuels, auditifs, kinésiques. (Myriam)

Varier les méthodes et approches en enseignement et introduire les aspects technologiques et multimodaux sont d'une grande aide pour les apprentissages des élèves. (Nora)

Dans la majorité des projets d'innovation, les participants ont fait preuve de créativité à toutes les étapes, soit lors de l'élaboration du projet d'innovation, du pilotage, des réajustements et de l'évaluation. Lors de la présentation finale des projets, nous avons noté chez les stagiaires un engagement manifeste, un investissement personnel (temps et moyens), une grande souplesse (adaptation à la situation), et ceci, malgré les contraintes des milieux d’accueil des stagiaires.

Notons aussi que, dans la partie «bilan » de projets d'innovation, les stagiaires ( $N=10)$ sont critiques quant aux usages diversifiés des outils multimodaux et à la gestion du temps.

Les bilans des dix stagiaires proposent aussi des pistes d'amélioration à leurs projets. Tous envisagent de reprendre leurs projets, mais soulignent le manque d'aide et de soutien technique.

Je pourrais le refaire en modifiant certaines équipes, en leur laissant le temps nécessaire pour réaliser le journal télévisé, possiblement leur faire confiance quant à filmer seuls dans l'école et sur son territoire extérieur, en changeant peut-être le cellulaire par un autre outil. (France)

\section{Les conditions pour la stabilisation des pratiques en LMM}

Lors des présentations finales des projets d'innovation et dans les bilans réflexifs, les stagiaires affirment avoir beaucoup appris sur leurs propres compétences en TIC et en LMM en contexte d'enseignement-apprentissage de leur discipline.

J'ai réalisé que j'étais davantage en mesure que je le pensais de concevoir des situations d'enseignement-apprentissage adaptées aux élèves et qui les intéressent. Aussi, intégrer les technologies n'a pas été un problème et même que j'ai trouvé que c'était beaucoup plus facile d'attirer l'attention des élèves. Je suis donc très fière de mon projet, même si je modifierais plusieurs choses si j'avais à le refaire. Il m’a permis de réaliser que je sous-estimais mes talents de création et que les projets de ce genre qui s'étalent sur plusieurs cours prennent beaucoup de temps à construire, mais qu'en fin de compte, ils sont très positifs et on gagne du temps à les faire.»(France) 
Durant le processus, j'ai réalisé certaines choses. Donc je vais modifier mon activité pour une prochaine fois. (Tania)

Comme nous le mentionnions plus haut, une difficulté majeure signalée par tous les stagiaires est le manque de collaboration et de soutien technique de la part des pairs et de certaines directions d'écoles (enseignants associés, professeurs ressources, personnel d'aide en technologie), et cela, en dépit du fait que la majorité des écoles qui ont accueilli les stagiaires étaient très bien dotées en termes de matériel et de locaux.

J'avais obtenu le soutien de quelques personnes pour faire les capsules. Malheureusement, au moment de filmer, pour le montage et la prise de son, plus personne n'était disponible. Je me suis retrouvé avec un éclairage médiocre et mon propre appareil photo numérique pour filmer. (Paul)

Les difficultés inhérentes aux usages de la technologique par des enseignants novices ont été rapidement compensées par les participants qui ont eu recours aux compétences en TIC et LMM de leurs propres élèves. On peut ainsi parler de co-construction des savoirs, dans un contexte hautement collaboratif, entre enseignants et élèves. Une des conditions de la stabilisation des pratiques d'intégration des TIC et de la LMM serait ainsi de concevoir une intégration des compétences informelles chez les élèves (ex. : maitrise technique des usages technologiques) dans l'élaboration même des scénarios didactiques avec l'enseignant.

Les réponses au posttest démontrent que malgré un sentiment de familiarité avec les TIC et les supports multimodaux, les participants ressentent une insécurité profonde à manipuler les modes et les supports en situation d'enseignement. Ainsi à la question : comment évaluez-vous votre compétence à utiliser la multimodalité dans vos cours?, les stagiaires ont répondu de la façon suivante: ".... oui mais, de façon restreinte et limitée » (p5) ; " Je me débrouille bien, cependant je suis moins à l'aise avec les cellulaires » (p9) ; "Je ne suis pas très à l'aise avec les outils » (p4). Les réponses aux questionnaires confirment leur réserve à devoir manipuler plusieurs outils technologiques et la LMM pour la production et l'édition vidéo ${ }^{19}$.

19. Voir figures 3, 4 et 7 pour la synthèse des résultats. 


\section{Conclusion}

L'avènement de nouvelles littératies qualifiées de multimodales auxquelles les apprenants ainsi que leurs enseignants sont de plus en plus confrontés pour accéder aux savoirs nous a amenées à réfléchir aux moyens d'intégrer la multimodalité et les TIC dans le champ de la didactique et dans celui de la formation des enseignants stagiaires. Actuellement, un grand nombre de ces jeunes enseignants stagiaires et les élèves auxquels ils font la classe sont des « digital natives » (Prensky, 2001) et se retrouvent forcément à manipuler des savoirs à l'aide de la multimodalité et des technologies. Cependant, à la lumière de notre recherche, on ne peut que déplorer une disparité et une pauvreté dans la formation effective à des usages harmonisés et efficaces de la technologie en classe, ce qui se remarque par les réticences et les craintes des participants à faire appel à la multimodalité pour innover dans leur enseignement des disciplines. Cela s'explique certainement par le manque de préparation des enseignants stagiaires en formation initiale, notamment dans les cours disciplinaires, et aussi par le cloisonnement de la compétence technologique dans les programmes de formation des maitres. De plus, nous attribuons aussi le peu de transfert de la recherche dans les pratiques enseignantes au manque de volonté des établissements scolaires qui n'ont pas encore pris la réelle mesure des recommandations émises par les recherches des dernières années sur la nécessité de soutenir les enseignants dans l'intégration des outils technologiques et multimodaux pour favoriser l'appropriation des savoirs disciplinaires. Ainsi, les enseignants qui s'essayent à des projets innovants se voient encore très peu encouragés, et les ressources mises à leur disposition sont déficitaires. Or, il apparait qu'une meilleure synergie entre la LMM, les TIC et les champs disciplinaires en formation initiale ouvre la voie à un renouvèlement des pratiques enseignantes en phase avec les recherches actuelles. Nous voyons ici des possibilités de collaborations plus étroites par le biais de la recherche-action-formation entre la recherche universitaire et la formation pratique à l'enseignement. Les avancées scientifiques viendraient ainsi soutenir les besoins des enseignants en formation identifiés à même leur expérience en classe. C'est une manière efficace de rendre les savoirs théoriques plus signifiants pour les enseignants en formation et peut-être de les motiver à poursuivre leur engagement dans la profession à travers la formation continue. 


\section{Références}

Barrette, C. (2004). Vers une métasynthèse des impacts des TIC sur l'apprentissage et l'enseignement dans les établissements du réseau collégial québécois. De la recension des écrits à l'analyse conceptuelle. Clic, 55, 8-15.

Béliveau, G. (2011). Analyse de recherches sur les TIC. Impact de l'usage des TIC au collégial. Rapport final. Département de philosophie du CÉGEP de Trois-Rivières. Québec. Repéré à http://philosophie.cegeptr.qc.ca/2011/08/analyse-des-recherches-sur-les-tice/

Bibeau, R. (2003). Taxonomie des contenus numérisés et des projets éducatifs avec les TIC. Direction des ressources didactiques, Ministère de l'Éducation du Québec. Repéré à http://eureka.ntic. org/file_download.php?lom_id=3033\&filename=taxonomie.pdf

Bussière, T. et Gluszynski, P. (2004). The impact of computer use on reading achievement of 15-yearolds. Learning Policy Directorate, Strategic Policy and Planning Branch Human. Resources and Skills Development. Canada.

Charlier, B. (2005). Parcours de recherche-action-formation. Revue des sciences de l'éducation, 31(2), 259-272.

Charlier, B. (2009). Réifier les pratiques d'enseignement: un enjeu de recherche et de formation. Dans F. Larose et A. Jaillet (dir.), Usages et mésusages de la trace informatique en enseignement et en recherche en éducation (p. 191-216). Paris : L'Harmattan.

Cope, B. et Kalantzis, M. (dir.). (2000). Multiliteracies. Literacy learning and the design of social futures, Londres : Routledge.

Dagenais, D. (2012). Littératies multimodales et perspectives critiques. Recherches en didactique des langues et des cultures: Les Cahiers de l'Acedle, 9(2), 5-46.

Foucher, A. L., (1998). Réflexions linguistiques et sémiologiques pour une écriture didactique du multimédia de la langue. ALSIC, 1(1) 3-25.

Gagnon, R. et Ziarko, H. (2011). Le texte informatif : un outil langagier pour apprendre. Québec français, 161, 50-52.

Gauthier, C. (2006). L'intégration des TIC dans les pratiques pédagogiques en milieu francophone minoritaire : tendances et défis, (collaboration de Mario Richard et Steve Bissonnette) : Fédération canadienne des enseignantes et des enseignants (FCE).

Gouvernement du Québec (2001). La formation à l'enseignement. Les orientations. Les compétences professionnelles. Québec : Ministère de l'Éducation.

Jewitt, C. (2002). The move from page to screen: the multimodal shaping of school. English. Visual communication, 1(2), 171-195. 
Koehler, M. J. et Mishra, P. (2008). Introducing TPACK. Dans AACTE Committee on Innovation \& Technology (dir.). Handbook of technological pedagogical content knowledge for educators (p. 3-29). New York : Routledge.

Kress, G. (2010). Multimodality: a social semiotic approach to contemporary communication. New York : Routledge.

Kress, G. et Van Leeuwen, T. (2001). Multimodal Discourses: The Modes and Media of Contemporary Communication. Londres: Edward Arnold.

Kress, G. et coll. (2001). Multimodal teaching and learning. The rhetorics of the science classroom. London (Continuum).

L'Écuyer, R. (1990). Méthodologie de l'analyse développementale des contenus. Méthode GPS et concept de soi. Québec: Presses de l’Université du Québec.

Lacelle, N., Lebrun, M., Boutin, J.-F., Richard M. et Martel, V. (2015). Les compétences en littératie médiatique multimodale au primaire et au secondaire: une grille d'analyse transdisciplinaire. Dans L. Lafontaine et J. Pharand (dir.), Littératie : vers une maitrise des compétences dans divers environnements (p. 168-184). Québec: Presses de l'Université du Québec,

Lebrun, M., Lacelle, N. et Boutin, J.-F. (2012a) (dir.). La littératie médiatique multimodale. De nouvelles approches en lecture-écriture à l'école et hors de l'école. Québec : Presses de l'Université du Québec.

Lebrun, M., Lacelle, N. et Boutin, J.-F. (2012b). Genèse et essor du concept de littératie médiatique multimodale. Dans Eli MacLaren (dir.), Mémoires du livre, Studies in Book Culture. Repéré à http://www.erudit.org/revue/memoires/2012/v3/n2/index.html

Loisier, J. (2011). Les nouveaux outils d'apprentissage encouragent-ils réellement la performance et la réussite des étudiants en FAD? Document préparé pour le Réseau d'enseignement francophone à distance du Canada. http://www.refad.ca/recherche/TIC/TIC_et_reussite_des_etudiants.pdf

Mishra, P. et Koehler, M. J. (2006). Technological Pedagogical Content Knowledge: A Framework for Teacher Knowledge. Teachers College Record, 108(6), 1017-1054.

Mishra, P., Koehler, M. et Harris, J. (2009). Teachers' Technological Pedagogical Content Knowledge and Learning Activity Types: Curriculum-based Technology Integration Reframed. Journal of Research on Technology in Education, 41(4), 393-416.

Herring, M. C., Mishra, P. et Koehler, M. J. (2016). Handbook of technological pedagogical knowledge (TPACK) for educators. New York: Routledge.

Prensky, M. (2001). Digital Natives, Digital Immigrants Part 1. (R. K. Belew et M. D. Vose, dir.) On the Horizon, 9(5), 1-6.

Prud'homme, L. (2007). La différenciation pédagogique: analyse du sens construit par des enseignantes et un chercheur-formateur dans un contexte de recherche-action-formation (Thèse de doctorat inédite). Université du Québec en Outaouais. 
Schmidt, D. A., Baran, E., Thompson, A. D., Mishra, P., Koehler, M. J. et Shin, T. S. (2009). Technological Pedagogical Content Knowledge (TPACK): The Development and Validation of an Assessment Instrument for Preservice Teachers. Journal of Research on Technology in Education, 42(2), 123149.

Shulman, L. S. (1986). Those who understand: Knowledge growth in teaching. Educational Researcher, 15(2), 4-14.

Street, B., Pahl, K. et Rowsell, J. (2009). Multimodality and New Literacy Studies. Dans J. Carey (dir.), The Routledge Handbook of Multimodal Analysis (p. 191-200). Londres : Routledge.

Van der Maren, J.-M. (2002). En quête d’une recherche pédagogique. Dans J. Donnay et M. Bru (dir.), Recherches, pratiques et savoirs en éducation (p. 89-104). Bruxelles : de Boeck et Larcier.

Viau, R. (2005). 12 questions sur l'état de la recherche scientifique sur l'impact des TIC sur la motivation à apprendre ». Université de Sherbrooke. Repéré à http://tecfa.unige.ch/tecfa/teaching/ LME/lombard/motivation/viau-motivation-tic.htm

Villeneuve, S., Karsenti, T., Raby, C. et Meunier, H. (2012). Les futurs enseignants du Québec sont-ils technocompétents? : une analyse en fonction de la compétence professionnelle à intégrer les TIC. Revue internationale des technologies en pédagogie universitaire, 9(1), 78-99.

Walsh, M. (2008). Worlds have collided and modes have merged: Classroom evidence of changed literacy practices. Literacy, 42(2), 101-108.

\section{Sitographie}

http://www.tpack.org/ 


\section{Annexes}

\section{Annexe 1}

Le tableau qui suit présente la liste des stagiaires et leurs projets par discipline enseignée.

\begin{tabular}{|c|c|c|c|}
\hline Projets & Discipline enseignée & Titre du projet & Classes et modalités de réalisation du projet \\
\hline Projet 1 & Français & L'histoire de l'humour & $\begin{array}{l}4 \text { classes de secondaire } 5 \text {. } \\
\text { En solo, en dyades et en grand groupe. }\end{array}$ \\
\hline Projet 2 & Français & Du populaire au littéraire & $\begin{array}{l}3 \text { groupes de secondaire } 2 . \\
\text { En équipes de } 4 \text { ou } 5 \text {. }\end{array}$ \\
\hline Projet 3 & Français & Les verbes pour conter & $\begin{array}{l}1 \text { classe de secondaire } 1 . \\
\text { En solo, en dyades et en grand groupe }\end{array}$ \\
\hline Projet 4 & Français & Les contes en rythme & $\begin{array}{l}1 \text { classe de secondaire } 3 . \\
\text { En solo, en dyades et en grand groupe. }\end{array}$ \\
\hline Projet 5 & Français & $\begin{array}{l}\text { La poésie et les jeux de mots de Marc Fa- } \\
\text { vreau et de Fred Pellerin }\end{array}$ & $\begin{array}{l}2 \text { groupes d'élèves de secondaire } 3 . \\
\text { En solo, en dyades et en grand groupe. }\end{array}$ \\
\hline Projet 6 & Sciences et technologie & L'anatomie humaine & $\begin{array}{l}1 \text { classe de secondaire } 3 . \\
\text { En science et technologie, en solo, en dyades et en } \\
\text { grand groupe }\end{array}$ \\
\hline Projet 7 & Univers social & Une image vaut mille mots & $\begin{array}{l}4 \text { groupes de secondaire } 1 . \\
\text { En solo. }\end{array}$ \\
\hline Projet 8 & Univers social & Les stars de la Renaissance & $\begin{array}{l}4 \text { groupes de secondaire } 2 . \\
\text { En solo, en dyades, autres modalités. }\end{array}$ \\
\hline Projet 9 & Univers social & Exposé des mouvements de pensée & $\begin{array}{l}6 \text { classes de secondaire } 4 . \\
\text { En solo, en dyades et en grand groupe. }\end{array}$ \\
\hline Projet 10 & Maths et physique & L'univers matériel en résumé. Maths & $\begin{array}{l}1 \text { classe de secondaire } 4 . \\
\text { En dyades. }\end{array}$ \\
\hline
\end{tabular}


Annexe 2

\section{Modalités de formation et de suivis des stagiaires}

\begin{tabular}{|c|c|c|c|}
\hline Encadrement & Durée/nombre & $\mathrm{N}$ & Objectifs \\
\hline $\begin{array}{l}\text { Séminaires de formation } \\
\text { à la multimodalité et à } \\
\text { ses usages pédagogiques } \\
\text { et didactiques. }\end{array}$ & $\begin{array}{l}4 \text { séminaires (durée de } 3 \text { h chacun) } \\
\text { séminaires et rencontres compre- } \\
\text { nant des cours magistraux et des } \\
\text { rencontres individuelles offerts aux } \\
\text { stagiaires entre janvier et avril } 2013 .\end{array}$ & $N=16$ & $\begin{array}{l}\text { Discussion des projets possibles avec chaque stagiaire. } \\
\text { Présentation des attentes du projet d'innovation et des contraintes } \\
\text { s'y rattachant. } \\
\text { Recherche d'idées et de ressources. } \\
\text { Modalités d'intégration de la multimodalité aux contenus à ensei- } \\
\text { gner. } \\
\text { Détermination de situations problèmes et conceptualisation des } \\
\text { projets. } \\
\text { Présentation du cadre de la multimodalité et formation à ses } \\
\text { usages dans le champ de la littératie et de ressources techno- } \\
\text { logiques, numériques en lien avec des usages pédagogiques } \\
\text { disciplinaires. }\end{array}$ \\
\hline Rencontres Skype & 3 rencontres d'environ une heure. & $N=1$ & $\begin{array}{l}\text { Demande d'aide et d'orientation pour le projet d'innovation. } \\
\text { Partage de difficultés inhérentes au contexte du stage (gestion de } \\
\text { classe, gestion de soucis liés aux ressources humaines et maté- } \\
\text { rielles, motivation des élèves.) } \\
\text { Adéquation des objectifs d'enseignements apprentissage avec les } \\
\text { attendus du projet d'innovation. }\end{array}$ \\
\hline Échanges courriel & 24 messages & $N=5$ & $\begin{array}{l}\text { Seulement } 5 \text { stagiaires ont sollicité de l'aide ou des éclaircisse- } \\
\text { ments par courriels. } \\
\text { Vingt-et-un (21) courriels concernant les projets d'innovation per- } \\
\text { sonnels de certains étudiants et trois (3) courriels plus généraux de } \\
\text { recadrage et d'explicitation des documents d'accompagnements } \\
\text { fournis aux stagiaires. }\end{array}$ \\
\hline
\end{tabular}


Annexe 3

\section{PLAN DU RAPPORT D’ANALYSE DU PROJET D’INNOVATION PÉDAGOGIQUE}

L'analyse de l'expérimentation d'un projet d'innovation pédagogique comportera les quatre (4) sections suivantes, chacune comprenant des étapes d'un processus de résolution de problèmes par l'innovation pédagogique.

\section{Compréhension du problème}

- Identification d'une problématique (identification d'un écart entre une situation observée et une situation désirée) et précision du problème (énoncé général);

- Description du problème (ses manifestations et ses principaux aspects);

- Recherche et localisation d'informations permettant d'y apporter une solution (courte recension d'écrits récents portant sur le problème et sa solution : minimum 3 sources);

- Objectifs pédagogiques ou didactiques poursuivis par le stagiaire.

\section{Intervention}

- Planification du projet en fonction du problème identifié et des éléments découlant de la recension des écrits;

- $\quad$ Planification des moyens envisagés pour vérifier les effets de l'intervention;

- Mise à l'essai du projet d'innovation pédagogique (description du contexte et des conditions de cet essai).

\section{Évaluation}

- Présentation des conséquences de l'intervention (quelles sont-elles?);

- Interprétation des conséquences de l'intervention (quels sont les liens entre l'intervention et ses conséquences?).

\section{Conclusion}

- Retour sur les objectifs poursuivis;

- Objectivation (apprentissages faits ou compétences développées par le stagiaire);

- $\quad$ Prospectives (réinvestissement possible dans des situations analogues). 


\section{Pour viser à réaliser un rapport de qualité, veillez à}

- la précision de l'énoncé du problème;

- la pertinence des écrits recensés;

- la précision de la planification du projet;

- la cohérence de l'intervention par rapport au contexte et aux écrits recensés;

- la clarté de la narration de l'intervention;

- l'originalité et au caractère novateur du projet;

- la précision des effets observés;

- la pertinence du jugement porté sur l'atteinte des objectifs du projet;

- la rigueur de la réflexion sur ses apprentissages;

- la richesse des pistes de réinvestissement;

- la qualité de la présentation matérielle du travail. 